Revue internationale de l'économie sociale

Recma

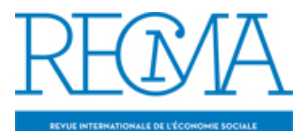

\title{
Des chantiers à venir pour la Recma
}

\section{Jean-François Draperi}

Numéro 288, avril 2003

URI : https://id.erudit.org/iderudit/1022192ar

DOI : https://doi.org/10.7202/1022192ar

Aller au sommaire du numéro

Éditeur(s)

Institut de l'économie sociale (IES)

ISSN

1626-1682 (imprimé)

2261-2599 (numérique)

Découvrir la revue

Citer ce document

Draperi, J.-F. (2003). Des chantiers à venir pour la Recma. Revue internationale de l'économie sociale, (288), 4-5. https://doi.org/10.7202/1022192ar d'utilisation que vous pouvez consulter en ligne.

https://apropos.erudit.org/fr/usagers/politique-dutilisation/ 


\section{DES CHANTIERS À VENIR POUR LA RECMA}

$\mathrm{N}$ ous publions dans ce numéro les résultats d'une enquête réalisée auprès des lecteurs de la Recma. Ce questionnaire avait pour but de mieux connaître leurs attentes et leurs avis sur plusieurs questions que le comité de rédaction, au-delà des activités qui lui incombent, se pose régulièrement. Ainsi, la spécialisation toujours plus grande des sciences économiques et sociales permet-elle encore de produire des textes susceptibles d'intéresser non seulement les spécialistes de chaque discipline - ce qui constitue l'objectif d'une revue scientifique classique -, mais aussi les collègues d'autres disciplines et plus encore les professionnels?

La question est d'autant plus présente que nous avons cherché, au cours des dernières années, à mieux garantir le niveau scientifique de la revue, en adoptant la double relecture de chaque soumission d'article selon le principe du " double aveugle ", c'est-à-dire en gardant l'anonymat réciproque des auteurs et des lecteurs. En règle générale, nous pensons qu'il est tout à fait possible de rester clair et lisible tout en faisant œuvre théorique. Nous privilégions simplement les articles qui articulent l'approfondissement théorique à un terrain concret. Par ailleurs, nous pensons que, au-delà des découpages disciplinaires, de nouvelles problématiques émergent en lien avec de nouvelles questions économiques et sociales. L'économie sociale est l'une d'entre elles, et elle justifie de questionner les découpages disciplinaires classiques et de contribuer à l'émergence de nouvelles disciplines.

Autre sujet récurrent : la langue de publication de la revue. La langue anglaise est devenue le langage dominant des rencontres internationales, même lorsque les congressistes connaissent une autre langue. L'une des raisons de cette suprématie réside dans le fait que la production et la diffusion des travaux anglo-saxons sont beaucoup plus importantes que nulle autre, et que leur audience internationale est incomparable. Quel avenir, dans ce contexte, pour une revue à vocation internationale de langue française? Le comité s'est toujours prononcé en faveur du maintien de la publication de la Recma en français, en particulier parce qu'il constate que l'adoption de la langue anglaise s'articule inévitablement à des choix théoriques (sauf à être en mesure de traduire les textes du français vers l'anglais, ce qui n'est pas le cas). Ouverte à tous les courants qui abordent les questions en lien avec l'économie sociale, solidaire, alternative, la démocratie économique, la question du " non-profit ", la solidarité, la mutualité, l'association, le développement durable, etc., la Recma est 
également héritière d'une longue histoire coopérative européenne et se reconnaît de ce fait préférentiellement dans une position théorique et une posture épistémologique originales, qui different sensiblement des problématiques de recherche les plus développées dans le monde anglo-saxon. L'enquête auprès de nos lecteurs nous conforte dans ce choix. Notre lectorat se répartit sur une bonne vingtaine de pays et la question des moyens du développement de l'audience internationale de la Recma n'en est pas moins posée.

Troisième sujet de débat: le comité perçoit la nécessité de réaliser un double déploiement de ses correspondants. Un déploiement géographique doit s'effectuer au niveau régional français. Nous devons complémentairement mieux nous appuyer sur l'excellent réseau international associé de longue date à la Recma. Ce déploiement géographique doit être couplé à un partenariat plus permanent avec les organisations de l'économie sociale, aussi bien au niveau régional qu'au niveau international. L'enquête confirme l'intérêt de réaliser ce travail.

Nous avions un quatrième sujet quasi permanent de débat : la création d'une collection d'ouvrages complémentaire à la revue. C'est fait : le premier livre est paru, d'autres suivront, et il n'est pas désagréable de clore une liste de problèmes par l'annonce de la résolution de l'un d'entre eux.

Jean-François Draperi 